\title{
EFFECTS OF GRADIENT MAGNETIC FIELD ON SWIRLING FLAME DYNAMICS
}

\author{
Inesa Barmina ${ }^{1}$, Maija Zake ${ }^{1}$, Uldis Strautins ${ }^{2}$, Maksims Marinaki ${ }^{2}$ \\ ${ }^{1}$ Institute of Physics, University of Latvia; \\ ${ }^{2}$ Institute of Mathematics and Computer Science, University of Latvia \\ mzfi@sal.lv, uldis.strautins@lu.lv
}

\begin{abstract}
The recent research was focused on experimental study and mathematical modelling of the gradient magnetic field effect on the development of swirling flame dynamics to better understand the mechanism of the interaction between the swirling flames and the non-uniform magnetic field and to provide control of the main gasification/combustion characteristics at thermo-chemical conversion of biomass (wood) pellets. The experimental study of the magnetic field influence on the swirling flow dynamics and on the composition of the products was carried out, using an experimental device, which is composed of a gasifier and water-cooled sections of the combustor, downstream of which the swirling flow field develops. The upper part of the gasifier was exposed to a transverse magnetic field. The magnetic field was created by two pairs of permanent magnets, producing a non-uniform, upstream increasing magnetic field with the mean axial magnetic field gradient $d B / d z \approx 0.8-1 \mathrm{~T} \cdot \mathrm{m}^{-1}$. The experimental study includes a joint research of the gradient magnetic field effect on the formation of flow velocity, flame composition and temperature profiles as well on the heat output at different stages of biomass thermo-chemical conversion providing analysis of the applicability of the gradient magnetic field for the control of biomass thermal decomposition, combustion of volatiles and heat energy production. The experimental results along with the mathematical modelling and computer simulation using different software confirm the influence of the external magnetic field on the stream, vorticity, temperature and species mass fraction fields indicating more effective burnout of volatiles and cleaner heat energy production.
\end{abstract}

Keywords: swirling flame, magnetic field, biomass, combustion characteristics.

\section{Introduction}

The influence of the magnetic field on combustion has been extensively studied with the aim to control the flame stability and combustion efficiency [1-3]. An experimental study of the diffusion flame response to the gradient magnetic field has shown that the flame structure and length can be controlled by varying the magnetic force, which acts on paramagnetic oxygen [1-3] by enhancing the axial and radial mass transfer of the flame species due to the magnetic wind effect along with the enhanced fuel-air mixing and enhanced fuel burnout.

The results of the experimental study and mathematical modelling have shown that, as a consequence of the magnetic field-induced Lorentz force in the flame, the magnetic field can affect the development of the swirling flame dynamics by increasing the local flow vorticity, which leads to enhanced fuel-air mixing with resulting variation of the local and average values of the flow velocity, temperature and composition [4]. Moreover, it was observed [5; 6] that the application of a gradient magnetic field to the swirling flame base when co-firing a fossil fuel (propane) with a renewable fuel (wood pellets) promoted the enhanced upstream air flow formation with the field-enhanced reverse axial mass transfer of paramagnetic oxygen up to the wood layer. With the increase of the thermal load into the primary reaction zone by co-firing the wood fuel with propane, the upstream air flow formation correlates with the field-induced increase of the reverse heat transfer by enhancing the thermal decomposition of wood pellets. Finally, this results in enhanced release and combustion of volatiles. In fact, the reverse field effect on the biomass thermal decomposition and on the combustion of volatiles can be attained provided the additional heat load is not used, and the reverse mass transfer of cold airflow results in cooling of the primary reaction zone by decreasing the rate of biomass thermal decomposition and the rates of the reaction rates.

Furthermore, the experimental study of the magnetic field effect on the magnetization of hydrocarbon fuels has shown that the action of the magnetic field on the hydrocarbon (HC) fuel flow can result in substantial changes in the fuel structure and combustion efficiency by reducing the fuel consumption in the engine [7;8]. This influence of the magnetic field on the combustion efficiency can be related to the formation of the isomeric form of the hydrocarbon atom with transition from the para-hydrogen state to a higher energized, more volatile, ortho-hydrogen state, thus attracting additional oxygen with direct influence on the combustion efficiency and on the composition of the products. 
Thus, the behaviour of the HC fuels and paramagnetic flame species under the influence of the magnetic field suggests an efficient method to control the fuel and air flow mixing, the combustion efficiency and the composition of emission. To better understand the magnetic field applicability for the control of the combustion characteristics developing downstream and upstream the swirling flame flow at thermal decomposition of wood fuel and combustion of volatiles, the main aim of the recent study was to provide complex experimental study and mathematical modelling of the processes developing under the influence of the magnetic field and to assess the dominant mechanism which causes improvement of the combustion characteristics.

\section{Experimental research}

The experimental study of the magnetic field influence on the swirling flow dynamics was carried out using a batch-size pilot device composed of a biomass gasifier and water-cooled sections of the combustor [6]. Discrete doses of biomass pellets (up to $280 \mathrm{~g}$ ) were supplied into the gasifier up to the inlet port of the propane flame flow that was used to initiate the wood fuel gasification and complete the burnout of the volatiles. The primary air at a rate of $201 \cdot \mathrm{min}^{-1}$ was supplied below the biomass pellets with the aim to initiate and sustain the biomass thermal decomposition, whereas the secondary swirling air at a flow rate up to $40 \mathrm{l} \cdot \mathrm{min}^{-1}$, which was supplied above the inlet port of the propane flame flow, was responsible for the mixing of the swirling air flow with the axial flow of the volatiles and for the burnout of the volatiles. The air supply rates in the device were controlled using Testo 6441 flowmeters.

The upper part of the gasifier was exposed to a transverse magnetic field induced by two pairs of permanent magnets (Fig. 1).

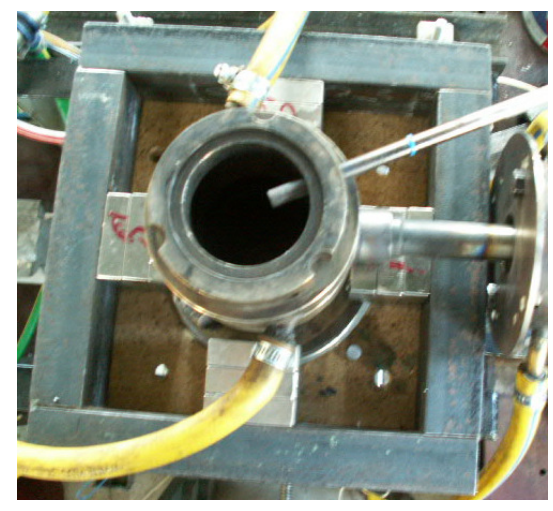

Fig. 1. Configuration of the device with application of permanent magnets to the upper part of the gasifier

The permanent magnets applied to the upper part of the gasifier produce a non-uniform magnetic field decreasing downstream along the primary reaction zone and developing close above the biomass layer, with the formation of axial and radial magnetic field gradients above and between the poles up to $d B / d z \approx 2.7-3.8 \mathrm{~T} \cdot \mathrm{m}^{-1}, d B / d r \approx 0.7-2.7 \mathrm{~T} \cdot \mathrm{m}^{-1}$ (Fig. $2-\mathrm{a}, \mathrm{b}$ ).
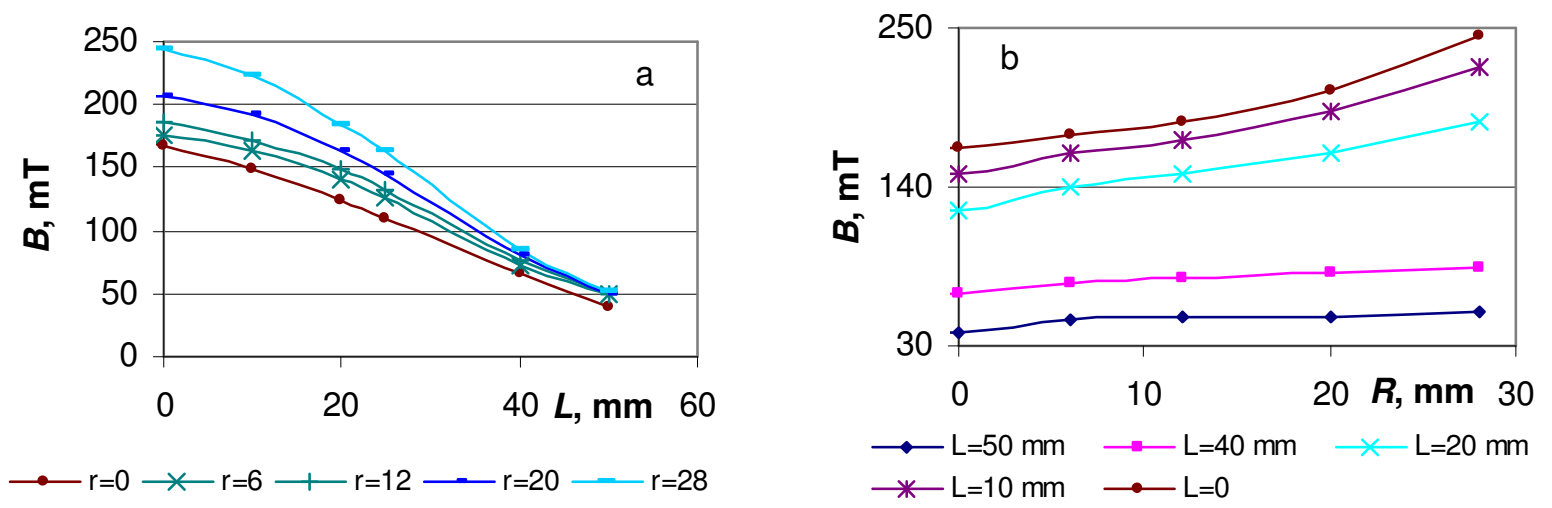

Fig. 2. Axial (a) and radial (b) distributions of the magnetic field induction 
The magnetic field action on the development of the swirling flame flow dynamics and on the formation of flame temperature and composition profiles was studied providing local measurements of the flow velocity, flame temperature and composition. The local measurements of the flame temperature were made using $\mathrm{Pt} / \mathrm{Pt}-\mathrm{Rh}$ thermocouples with data online registration by the Pico logger. The local measurements of the flame composition - mass fraction of the volatiles $\left(\mathrm{CO}, \mathrm{H}_{2}\right)$, volume fraction of the main product $\left(\mathrm{CO}_{2}\right)$, air excess ratio $(\alpha)$ and combustion efficiency - were made using a gas sampling probe and a Testo 350 gas analyzer. Calorimetric measurements of the cooling water flow were made to assess the impact of the inlet conditions on the heat output of the device and on the average values of the produced heat energy at different stages of biomass thermo-chemical conversion. The calorimetric measurements of the cooling water flow involve joint measurements of the cooling water mass flow and of the temperature at the inlet and outlet of each water-cooled section by AD 560 thermo sensors, with online data registration using the Data Translation DT9805 data acquisition module and the Quick DAQ program.

\section{Results and discussion}

The experimental investigations of the magnetic field effects on the swirling flame flow behaviour and on the main combustion characteristics were conducted at thermo chemical conversion of wood pellets by comparing the main combustion characteristics of the undisturbed flame flow $(B=0)$ and of the flame, which was subjected to the magnetic field $(B)$. First of all, the magnetic field disturbs the flame flow structure, which is noticeable (visible) if the field is applied to the free flame base. If the magnetic field is not applied to the flame, the ignition and flaming combustion of the volatiles occur at $t<700 \mathrm{~s}$, with the transition to the after-flame smouldering stage at $t>750 \mathrm{~s}$ (Fig. 3, a-d). If the magnetic field of the configuration as in Fig. 1 is applied to the free flame base, the magnetic force $(F \approx u x B)$ acts on the axial flow of the volatiles and flame ions $\left(\mathrm{CHO}^{+}, \mathrm{H}_{3} \mathrm{O}^{+}, \mathrm{C}_{2} \mathrm{H}_{3} \mathrm{O}^{+}, \mathrm{C}_{2} \mathrm{H}_{2}{ }^{+}, \mathrm{C}_{3} \mathrm{H}_{3}{ }^{+}\right)$ with a noticeable deformation of the free flow structure during the primary stage of swirling flame flow formation $(t<750 \mathrm{~s})$ and splits the flow into separate branches (Fig. 3-e, f). With further development of the flame reaction zone $(t>700 \mathrm{~s})$, the separate branches gradually merge (Fig. 3-g, h) promoting the field-induced transition to flaming combustion of the volatiles that was observed at $t \approx 800-850 \mathrm{~s}$.
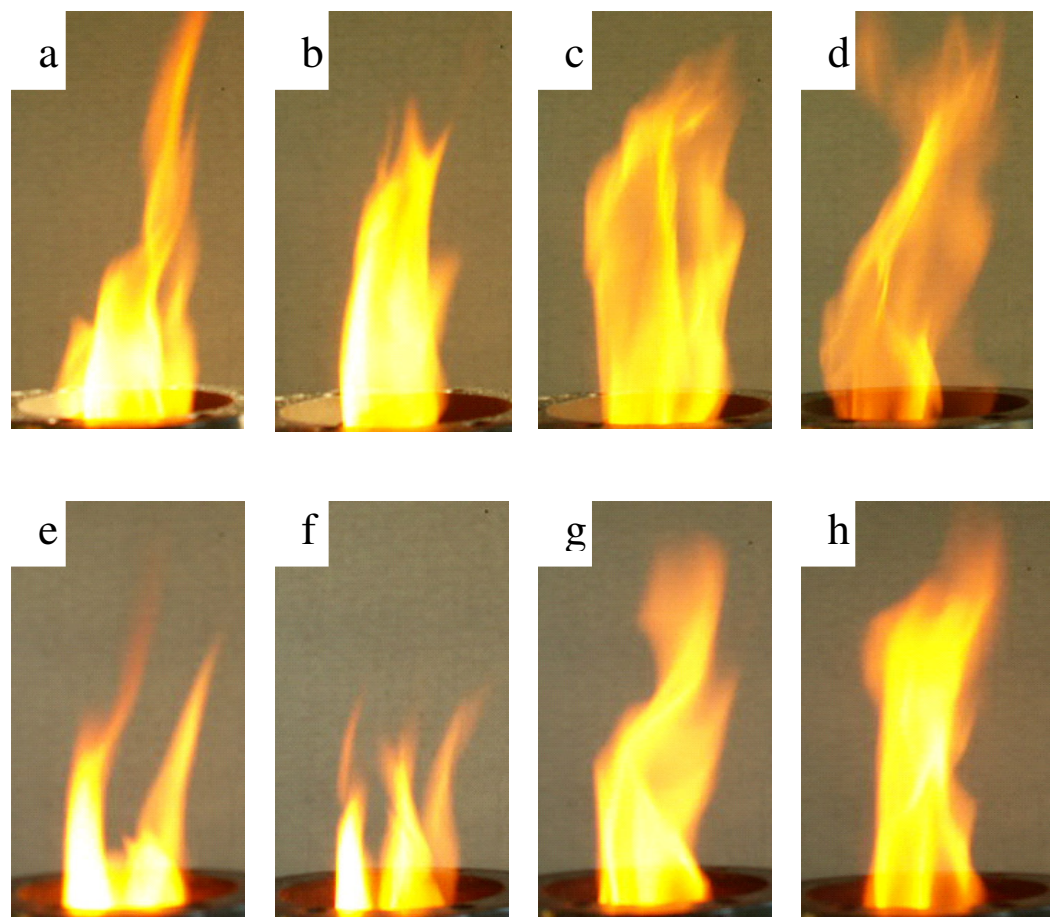

Fig. 3. Formation of the free flame length and shape at thermo-chemical conversion of wood pellets for the undisturbed flame flow $(B=0),(a-d)$ and for the flame subjected to the magnetic force (e-h): $\mathrm{a}, \mathrm{e}-t=650 \mathrm{~s} ; \mathrm{b}, \mathrm{f}-t=700 \mathrm{~s} ; \mathrm{c}, \mathrm{g}-t=750 \mathrm{~s} ; \mathrm{d}, \mathrm{h}-t=850 \mathrm{~s}$ 
The magnetic field-induced deformation of the flow structure at the primary stage of swirling flame flow formation evidences of the influence of the swirling flow velocity profiles on the formation (Fig. 4-a, b). The main field-induced deformation of the axial and tangential velocity profiles occurs in the outside part of the flame reaction zone $(r / R>0.5)$, where the pronounced decrease of the axial flow velocity $\left(u_{z}\right)$ correlates with the local increase of the tangential velocity $\left(u_{\varphi}\right)$ and of the flow swirl number near the water-cooled channel walls, which leads to the field-enhanced mixing of the flame components along the outside part of the flame reaction zone.

It should be emphasized that the average values of the axial and tangential velocities decrease under the influence of the magnetic field on the flow field. The average value of the axial flow velocity decreased from $0.76 \mathrm{~m} \cdot \mathrm{s}^{-1}$ to $0.65 \mathrm{~m} \cdot \mathrm{s}^{-1}$ and the average value of the tangential flow velocity decreased from $0.55 \mathrm{~m} \cdot \mathrm{s}^{-1}$ to $0.47 \mathrm{~m} \cdot \mathrm{s}^{-1}$ with the nearly constant value of the flow swirl number $(S \approx 0.48)$. The decrease of the average value of the axial flow velocity suggests that under the influence of the magnetic field on the swirling flame flow field the residence time of reactions $\left(t \approx 1 / u_{z a v}\right)$ developing at the thermo-chemical conversion of the combustible volatiles $\left(\mathrm{CO}, \mathrm{H}_{2}\right)$ increases, with their more complete combustion downstream the flame reaction zone.
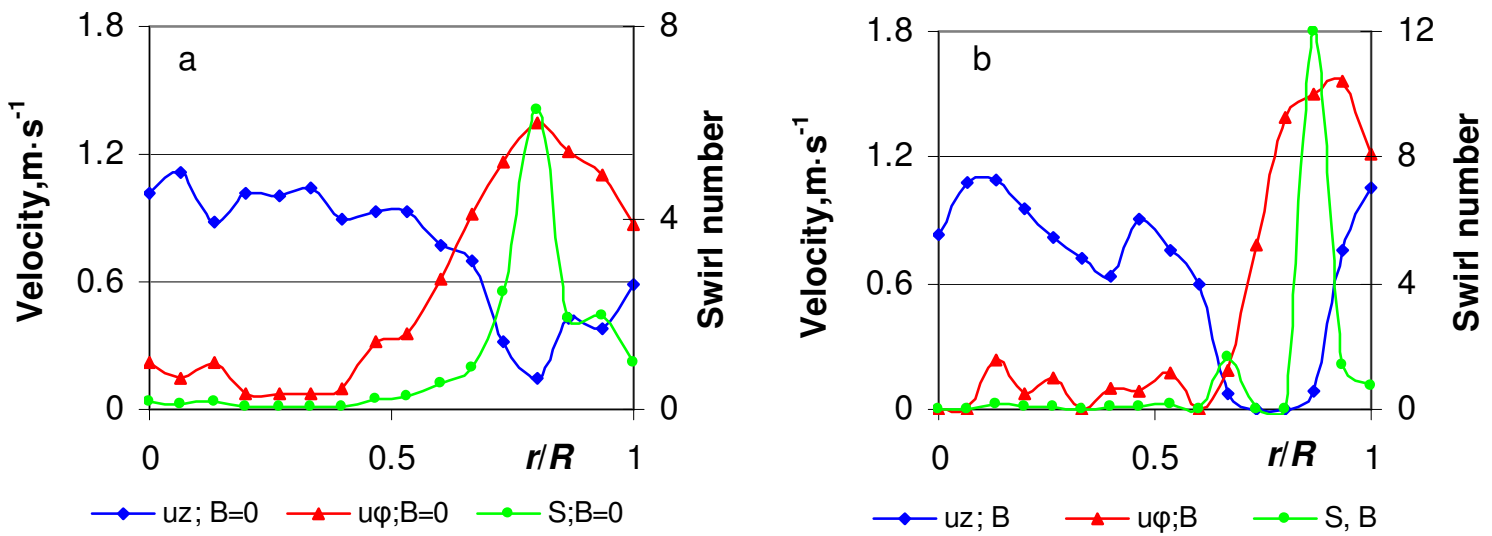

Fig. 4. Formation of the flow velocity profiles and swirl intensity of the undisturbed flame flow $(B=0)$ and of the flame flow which is subjected to the magnetic field

The field-enhanced combustion of the volatiles is confirmed by the complex measurements of the weight loss rate of the biomass (wood) pellets, of the heat output from different parts of the flame reaction zone, of the total amount of the heat output at thermo-chemical conversion of biomass pellets (Fig. 5-a, b), of the composition of the products and of the combustion efficiency (Fig. 6-a, b).
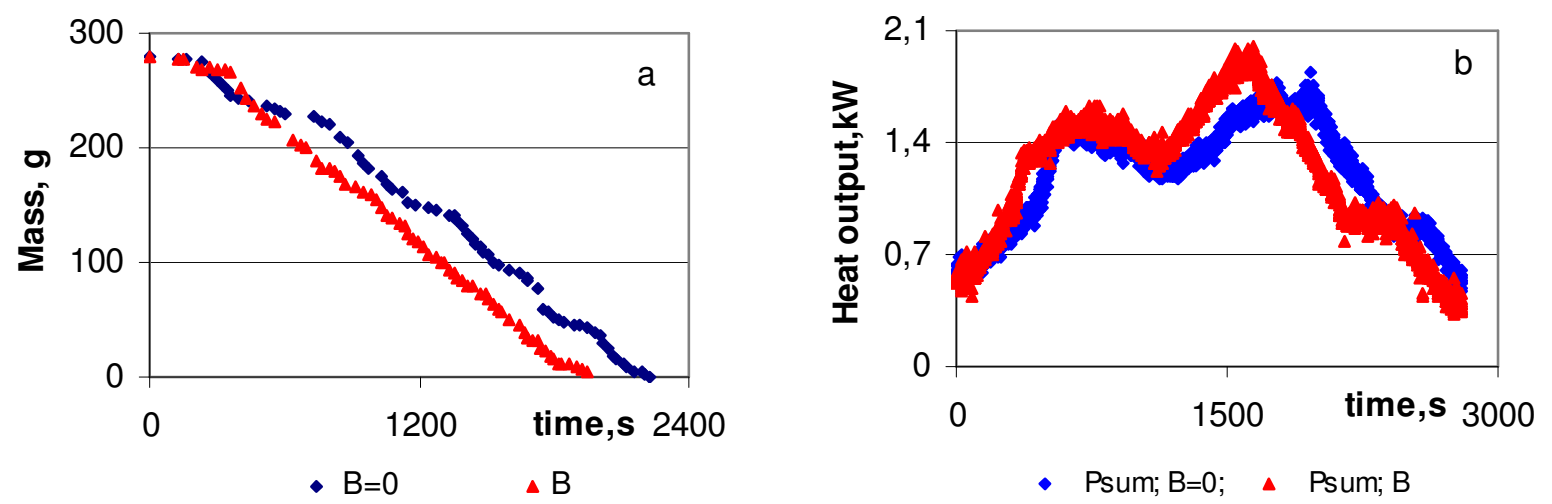

Fig. 5. Magnetic field effect on the biomass weight loss rate (a) and on the total amount of the heat output from the flame reaction zone (b) at thermo-chemical conversion of wood pellets

The average weight loss rate under the influence of the magnetic field increased from $0.13 \mathrm{~g} \cdot \mathrm{s}^{-1}$ up to $0.167 \mathrm{~g} \cdot \mathrm{s}^{-1}$, whereas the total amount of the heat output increased by about $8.5 \%$. In fact, the dominant field-enhanced increase of the heat output (up to19\%) was observed in the gasifier, which is 
placed below the swirling air nozzle. This suggests that the gradient magnetic field promotes the enhanced reverse mass transfer of paramagnetic oxygen up to the surface of the wood layer advancing so the faster ignition and combustion of the volatiles.

As a result of the field-enhanced combustion of the volatiles, the $\mathrm{CO}_{2}$ volume fraction in the products reduced, decreasing the mass fraction of the volatiles in the products (Fig. 6-a, b), which was observed during the flaming combustion of the volatiles as well as during the after-flame smoldering stage. Moreover, slightly (by about $3 \%$ ) the combustion efficiency increased confirming the improved combustion of the volatiles.
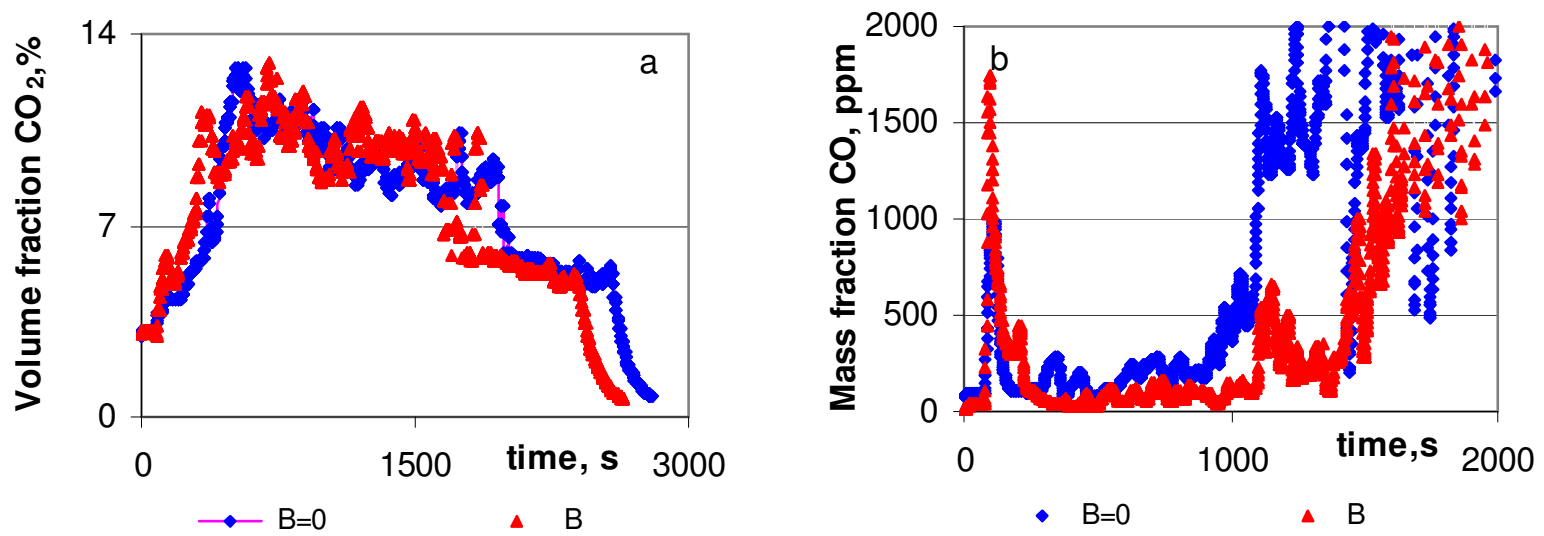

Fig. 6. Magnetic field effect on the time-dependent variations of the flame composition at thermo-chemical conversion of wood pellets

\section{Mathematical modelling and numerical results}

For mathematical modelling, we considered a system of partial differential equations describing an axially symmetric compressible reacting swirling flow and the temperature with a simple chemical reaction in a cylindrical pipe-combustor with the radius $r=0.05 \mathrm{~m}$, length $z=0.1 \mathrm{~m}$. This model is similar to $[9 ; 10]$, but has a different form of the radial $\left(F_{r}\right)$ and axial $\left(F_{z}\right)$ components of the Lorentz force. The goal was to provide a qualitative difference between the velocity distributions (considering the stream function $\Psi$ ) in cases when the external magnetic field induced by a permanent magnetic material wrapped around the computational domain was (or was not) applied. Simulations were made using the Matlab and FEMM software simultaneously [11].

The FEMM software provides a finite element solution of the Maxwell equations formulated for the magnetostatic case in two spatial dimensions [11]:

$$
\nabla \times H=0, \nabla \cdot B=0, B=\mu(H+M),
$$

where $H$ is the magnetic field intensity;

$B$ is the magnetic flux density;

$M$ stands for magnetization;

$\mu$ is the permeability constant.

The software considers the vector potential formulation in the case of two dimensions. Zero vector potential boundary condition was applied to the symmetry part of the boundary for the case of axial symmetry. For magnetic flux density, two magnetization directions, perpendicular to the flow direction and aligned with the flow direction, were considered (see Figs. 7, 8). The flux density in Figs. 7,8 varies between $0.001 \mathrm{~T}$ and $0.1 \mathrm{~T}$. For the calculations, a mean value of $0.03 \mathrm{~T}$ was considered.

After the $B$ field with the two components $B r, B_{z}$ is obtained, we use the Matlab built-in differentiation commands to calculate the Lorentz force terms in the following form:

$$
F_{r}=\left(\frac{\partial B_{r}}{\partial z}-\frac{\partial B_{z}}{\partial r}\right) B_{z}, F_{z}=-\left(\frac{\partial B_{r}}{\partial z}-\frac{\partial B_{z}}{\partial r}\right) B_{r} .
$$


For the inviscid flow approximation, we used the implicit FDS in time with upwind differences in space. To solve the discrete problem, we applied the ADI method of Douglas and Rachford, similarly as in $[9 ; 10]$.

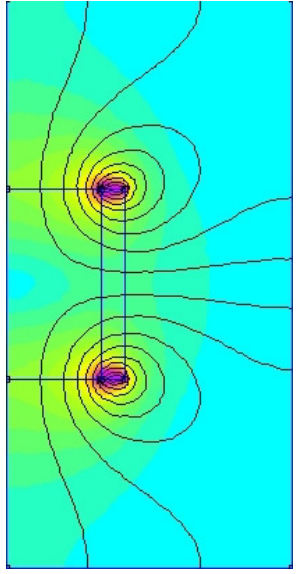

Fig. 7. Magnetic flux density plot: the magnetization direction perpendicular to the flow direction

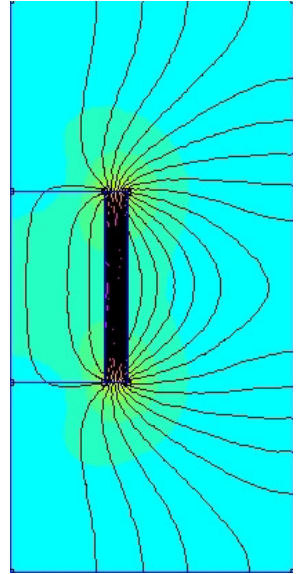

Fig. 8. Magnetic flux density plot: the magnetization direction aligned with the flow direction

Here some graphs are presented, which allows us to compare the stream function distributions when the source term is applied and not applied. One can see the counter-clockwise vortex rotation in Fig. $9(\Psi>0.125)$ an additional clockwise rotating vortex in Fig. $10(\Psi<0)$ and the disappearance of vorticity in Fig. 11.

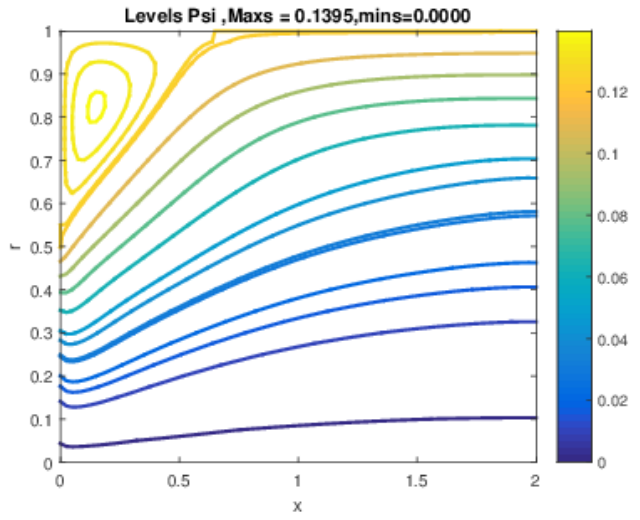

Fig. 9. Stream function, no magnetic field applied

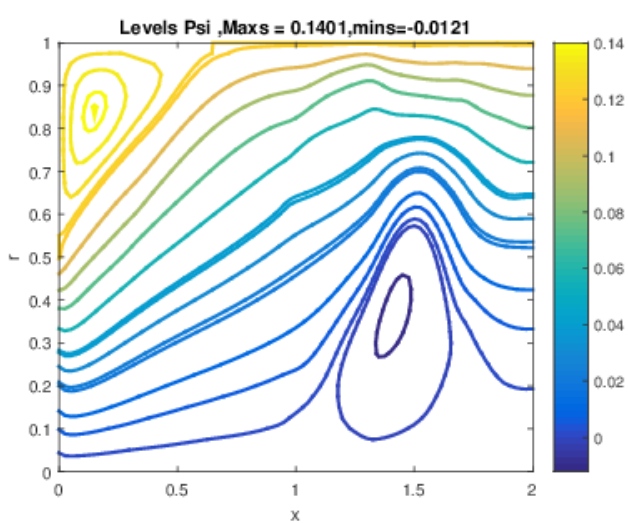

Fig. 10. Stream function; the magnetization direction perpendicular to the flow direction

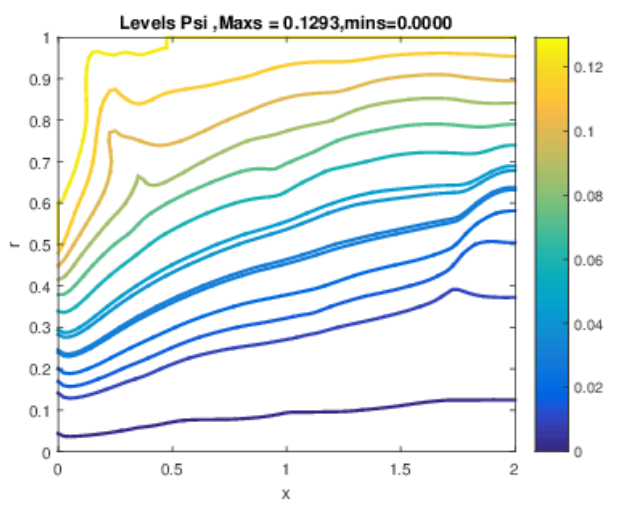

Fig. 11. Stream function; the magnetization aligned with the flow direction 


\section{Conclusions}

From the results presented above the following conclusions can be drawn:

1. The application of the magnetic field to the swirling flame flow disturbs the flow structure with direct influence on the formation of flow velocity profiles decreasing so the average values of the axial and tangential velocities and increasing the residence time of the reactions at thermochemical conversion of the volatiles.

2. Increasing the residence time of reactions downstream the flame reaction zone results in increase of the total heat output from the flame with the correlating increase of the $\mathrm{CO}_{2}$ volume fraction in the products and with the correlating decrease of the mass fraction of the volatiles.

3. The mathematical modelling has shown that the application of an external magnetic field induced by permanent magnets affects the velocity distributions and does not affect much the temperature and the concentration distribution if we stay at the chosen model bounds. The effect of magnetization perpendicular to the flow direction results in appearance of an additional vortex in the computational domain and in disturbance of the stream lines. The influence of magnetization aligned with the flow direction results in removal of the vortex in the upper left corner and in disturbance of the stream lines.

\section{Acknowledgements}

The authors would like to acknowledge financial support from the Latvian Research Cooperation Project of the Latvian Council of Science (No. 623/2014).

\section{References}

4. Gilard V., Gillon P., Blanchard C.J.N., Sarh B. Influence of a horizontal magnetic field on a coflow methane/air diffusion flame, Combustion Science and Technology, the stabilization of a lifted diffusion flame. Proc. the European Combustion Meeting, Vol. 180, 2009, pp.1920-1935.

5. N. I. Wakayama, Behavior of Gas Flow under Gradient Magnetic Fields, Journal of Applied Physics, Vol. 69, No. 4, February 1991, pp. 2734-2736

6. Sumathi Swaminathan, Effects of Magnetic Field on Micro Flames, A thesis for the degree of Master of Science in Mechanical Engineering, Louisiana State University, pp.117, 2005, http://etd.lsu.edu/docs/available/etd-11182005-092209/unrestricted/Swaminathan_thesis.pdf

7. Barmina I., Valdmanis R., Zake M., Kalis H., Marinaki M., Strautiņš, U. Magnetic field control of combustion dynamics. Latvian Journal of Physics and Technical Science, 2016, Vol. 53, N4, pp. 36-47.

8. Zake M., Barmina I., Bucenieks I., Krishko V. Magnetic field control of combustion dynamics of the swirling flame flow. Magnetohydrodynamics, Vol. 46, No.2, 2010, pp.171-186.

9. Barmina I., Bucenieks I., Gedrovics M., Krishko V., Zake M. The magnetic field effect on the swirling combustion of renewable fuel. Environmental and climate technologies, RTU, Vol. 3, ser. 13, 2009, pp. 11-17.

10. Ali A. Jazie Al-Khaledy. High performance and low pollutant emissions from a treated diesel fuel, using a magnetic field, Al-Qadisiya Journal for Engineering Sciences, Vol. 1, No. 2, 2008, pp. 211-224.

11. Chaware K. Review on effect of fuel magnetism by varying intensity on performance and emission of single cylinder four stroke diesel engine. International Journal of Engineering Research and General Science, Vol. 3, issue 1, 2015, pp. 1174-1178.

12. Kalis H., Barmina I., Zake M., Koliskins A. Mathematical Modelling and Experimental Study of Electrodynamic Control of Swirling Flame Flows, Engineering for Rural Development, pp. 134-141, 2016.

13. Kalis H., Marinaki M., Strautins U., Lietuvietis O. On the numerical simulation of the combustion process with simple chemical reaction, Proc. the 7-th Baltic Heat Transfer conf. "Advances in Heat Transfer", 175-180, August 24-26, 2015.

14. David Meeker. Finite Element Method Magnetics, Version 4.2, User's Manual, 2015. 